\title{
A Tight-Binding Study of Acceptor Levels in Semiconductors
}

\author{
J. G. Menchero \\ Instituto de Física, Universidade Federal do Rio de Janeiro \\ Cx. Postal 68.528, 21945-970 Rio de Janeiro, Brazil
}

Received February 8, 1999

\begin{abstract}
Acceptor binding energies in zinc-blende semiconductors are determined within the tight-binding formalism. The importance of fitting the valence-band masses in the (100) as well as (111) directions is discussed, and parametrizations that specifically fit the valence-band anisotropy are used to calculate Ge acceptor levels in $\mathrm{Al}_{x} \mathrm{Ga}_{1-x}$ As alloys. The sensitivity of the calculated energies to the parameters that determine bulk masses is investigated, as well as the effect of varying the on-site energy of the impurity. A comparison is made between first-neighbor and second-neighbor hopping models. For shallow levels, both approaches give the same results. For deeper levels, however, important differences arise. Experimental evidence suggests that first-neighbor models are better suited for describing intermediate to deep levels.
\end{abstract}

\section{Introduction}

The effective mass theory (EMT) has long been a principal tool for investigating shallow impurity states in semiconductors [1Г 2]. In EMTT it is assumed that the impurity wave function is highly delocalized in real space $\Gamma$ which in turn implies a strong localization in $k$ space. As a result The electronic properties of the host material may be described by only a few parameters related to the dispersion near the $k$ point in consideration. For instance Tin the Luttinger-Kohn version of $\mathbf{k} \cdot \mathbf{p}$ theory the electronic structure of the host is completely defined in terms of the spin-orbit energy and the three Luttinger parameters (LP's) $\Gamma$ which also determine the impurity energy level. Because each host material has a different set of LP'sTand acceptor binding energies are known to vary widely from host to host $\Gamma$ it is clear that the impurity level within EMT must depend sensitively on these bulk parameters.

Another result of EMT is that $\Gamma$ for a given materialTany singly ionized acceptor should have the same binding energy regardless of the species. HoweverTexperimentally it is known that such energies can vary widely from impurity to impurity. For exampleTan In acceptor in Si has a binding energy of 157 meVTcompared to $45 \mathrm{meV}$ for a $\mathrm{B}$ acceptor in the same host [3]. Such deviations from EMT are attributed to centralcell effects $\Gamma$ and demonstrate that the binding energies can also be very sensitive to the details of the potential in the immediate neighborhood of the impurity.

Recently an approach was presented [4] for calculating impurity states in semiconductors based on the tight-binding (TB) formalism. The localized basis set of TB provides a natural description for deep levels $\Gamma$ while the highly delocalized shallow levels are treated by means of very large unit cells together with a scaling law that allows extrapolation to the bulk limit [4]. In other words $T$ the TB approach is not intrinsically restricted to either the shallow or deep limits. For the TB approach to be usefulThowever Ta clear understanding of how the calculated energies depend on the TB parameters is required. In particular $\Gamma$ the sensitivity of EMT on the LP's and the the role of central-cell effects must be understood in the context of the current model. Another important question particular to TB concerns differences between first-nearest neighbor (1nn) and second-nearest neighbor (2nn) descriptions of the impurity state.

The aim of this paper is to carefully investigate how the impurity binding energies depend on the TB parameters that are most physically relevant to the impurity problem. The paper is organized as follows: In Sec. II the TB approach for calculating impurity states in semiconductors is briefly reviewed. In Sec. III the dependency of the impurity binding energy on the bulk 
effective masses and the on-site energy of the impurity is investigated. Comparisons are also made between $1 \mathrm{nn}$ and $2 \mathrm{nn}$ TB approaches with important differences found between the two. To illustrate these effects $\Gamma \mathrm{Ge}$ acceptors in GaAsT AlAsT and $\mathrm{Al}_{x} \mathrm{Ga}_{1-x}$ As alloys are considered. The summary and conclusions are given in
Sec. IV.

\section{Calculational Details}

Our TB Hamiltonian contains terms describing the bulk material as well as the impurityГand is given by [4]

$$
H=\sum_{i j} \sum_{\mu \nu} h_{\mu \nu}^{i j} c_{i \mu}^{\dagger} c_{j \nu}+\sum_{i} \sum_{\mu \nu} \lambda_{i}\langle\mu|l \cdot s| \nu\rangle c_{i \mu}^{\dagger} c_{i \nu}+\sum_{i} \sum_{\mu} U\left(r_{i}\right) c_{i \mu}^{\dagger} c_{i \mu},
$$

where the roman indices denote the site and the greek indices label the spin orbitals. The $h_{\mu \nu}^{i j}$ define all the on-site energies and hopping for the bulk material. In this work $\Gamma$ both $1 \mathrm{nn}$ and $2 \mathrm{nn}$ hopping are considered. The strength of the spin-orbit interaction for atom $i$ is defined by $\lambda_{i} \Gamma$ and the on-site energy for atom $i$ due to the Coulomb potential of the impurity is given by $U\left(r_{i}\right)$. For our basis set the $s p^{3} s^{*}$ orbitals proposed by Vogl [5] is usedrbut spin is included here leading to a total of 10 basis states per site.

The perturbation potential $U\left(r_{i}\right)$ is described by an isotropic $q$-dependent screening $\Gamma$

$$
U\left(r_{i}\right)=\frac{e^{2}}{\epsilon_{0} r_{i}}+A \frac{e^{2}}{r_{i}} e^{-\alpha r_{i}}+(1-A) \frac{e^{2}}{r_{i}} e^{-\beta r_{i}}-\frac{e^{2}}{\epsilon_{0} r_{i}} e^{-\gamma r_{i}}
$$

where the screening parameters $\mathrm{A} \Gamma \alpha \Gamma \beta \Gamma$ and $\gamma$ are taken from Bernholc [6]. Near the originTthe potential looks like a bare Coulomb potential $U_{\text {bare }}=e^{2} / r_{i} \Gamma$ but far away the potential looks like a bulk-screened potential $U_{\text {bulk }}=e^{2} / \epsilon_{0} r_{i}$ Twith $\epsilon_{0}$ being the static dielectric constant for the host material. Precisely at the impurity site $\left(r_{i}=0\right) \Gamma E q .(2)$ is undefined and the perturbation potential is assigned a value $U_{0} \Gamma$ where $U_{0}$ is a parameter describing central-cell effects.

In order to determine the acceptor energy first the energy $E_{v}$ at the top of the valence band is calculated for the pure system energy is easily found with the aid of Bloch's theorem. Next $\Gamma$ a single impurity is placed in a very large cubic supercell containing $8 L^{3}$ atoms arranged in the zincblende structure and subject to periodic boundary conditions $\Gamma$ with $L$ being the length of the supercell side in units of the conventional lattice parameter. The presence of the impurity breaks translational symmetry and introduces states in the gap region with energy above $E_{v}$. The energy $\tilde{E}_{v}(L)$ of the highest of these states determines the acceptor energy for supercell size $L$ via the relation $E(L)=\tilde{E}_{v}(L)-E_{v}$. The main task therefore is to calculate $\tilde{E}_{v}(L) \Gamma$ which is accomplished here using a variational algorithm that minimizes the expectation value of $\left\langle\Psi\left|\left(H-E_{\text {ref } f}\right)^{2}\right| \Psi\right\rangle$ Twith $E_{\text {ref }}$ being a reference energy suitably chosen near $\tilde{E}_{v}(L)[7]$. The computation time using this scheme scales linearly with the number of statesTmaking solutions possible even for very large supercells. For instance $\Gamma$ system sizes ranging up to $L=20$ (64000 atoms) are handled routinely [4]. However Tusing a recently proposed scaling law [4] $\Gamma$ the impurity binding energy can be extrapolated to the bulk limit using far smaller supercell sizes. In this scaling law Tthe energy $E(L)$ as a function of supercell size $L$ is given by

$$
E(L)=E_{a}+P e^{-\alpha L},
$$

with $E_{a}$ being the acceptor energy in the bulk limit $(L \rightarrow \infty) \Gamma$ and $P$ being a constant independent of $L$. The acceptor energy for the infinite system can therefore be found by calculating $E(L)$ for three relatively small supercells and then solving for $E_{a} \Gamma P \Gamma$ and $\alpha$ in 
Eq. (3). When using Eq. (3) to find the binding energy in the bulk limitTcaution must be exercised to verify not only that the supercell sizes are in the scaling regime $\Gamma$ but also that the scaled energies have converged to the limiting value.

\section{Results}

In EMTГ the acceptor binding energy depends on the three Luttinger parameters $\gamma_{1} \Gamma \gamma_{2} \Gamma$ and $\gamma_{3}$. These $\Gamma$ in turnTcan be related to the heavy-hole $(\mathrm{HH})$ and lighthole (LH) masses along (100) and (111) [8]

$$
\begin{aligned}
\gamma_{1} & =\frac{1}{2}\left[m_{l h}^{-1}(100)+m_{h h}^{-1}(100)\right], \\
\gamma_{2} & =\frac{1}{4}\left[m_{l h}^{-1}(100)-m_{h h}^{-1}(100)\right], \\
\gamma_{3} & =\frac{1}{4}\left[m_{l h}^{-1}(111)-m_{h h}^{-1}(111)\right],
\end{aligned}
$$

where the masses in these expressions and throughout this work are in units of the free-electron mass. The fact that the LP's depend on the valence-band masses in both the (100) and (111) directions suggests that both are very important to fit properly in the TB parametrizations. On the other hand $\Gamma$ due to the high density of states associated with the $\mathrm{HH}$ band $\Gamma$ these masses are expected to be much more important than the LH masses for the purposes of determining the acceptor binding energies. Tight-binding parametrizations specifically chosen to fit the HH masses have been determined [9] both for $1 \mathrm{nn}$ and $2 \mathrm{nn}$ models $\Gamma$ and will be used in this work. In Table 1 the valence-band masses resulting from these parametrizations are shown for GaAs and AlAsTtogether with the experimental [10] and theoretical [11] values.

The Ge acceptor in $\mathrm{Al}_{x} \mathrm{Ga}_{1-x}$ As alloys acts as a simple substitutional impurity at the As site. The binding energy has been measured experimentally [12] using photoluminescence in the direct-gap range $0<x<0.4 \Gamma$ and the results are given by the dotted line in Fig.1. For pure GaAsT the binding energy is $\sim 40$ meVTbut increases rapidly with an upward curvature to a relatively deep $\sim 120 \mathrm{meV}$ by $x=0.40$. The binding energy curves $E_{a}(x)$ calculated using the $1 \mathrm{nn}$ and $2 \mathrm{nn}$ parametrizations are given in Fig.1 by the solid and broken linesTrespectively. The VCA was used to obtain the Hamiltonian matrix elements for the alloy. NoteTof course $\Gamma$ that the VCA has no effect on the calculated binding energies at $x=0$ or $x=1 \Gamma$ which correspond to pure GaAs and pure AlAs respectively. The impurity perturbation potential $U_{0}$ was chosen in order that the calculated acceptor energy match the experimental value for pure GaAs. This led to a value of $3.42 \mathrm{eV}$ for $2 \mathrm{nn}$ and $2.80 \mathrm{eV}$ for $1 \mathrm{nn}$. These values of $U_{0}$ were then used for all $x$ Tso that any variation in the acceptor energy with respect to $x$ is attributable to the electronic response of the host material $\Gamma$ and not to central-cell effects. The result for $2 \mathrm{nn}$ is in qualitative agreement with experiment showing an increasing binding energy with increasing $\mathrm{Al}$ content. However $\Gamma$ it significantly underestimates the binding energy at $x=0.4$ Wwith an energy of $71 \mathrm{meV}$ compared to the experimental value of $120 \mathrm{meV}$. The result is improved considerably with $1 \mathrm{nn} \Gamma$ giving excellent agreement up to $x=0.2$. However Tthe results diverge beyond that $\Gamma$ and the binding energy of $87 \mathrm{meV}$ at $x=0.4$ is still substantially below the experimental value.

Table 1 - Heavy- and light-hole effective masses along [100] and [111] Tgiven in units of the free-electron mass.

\begin{tabular}{lccccc} 
Reference & Compound & $M_{H H}[100]$ & $M_{L H}[100]$ & $M_{H H}[111]$ & $M_{L H}[111]$ \\
\hline Experiment $^{a}$ & GaAs & 0.340 & 0.094 & 0.750 & 0.082 \\
2nn (this work) & GaAs & 0.346 & 0.074 & 0.751 & 0.066 \\
1 nn (this work) & GaAs & 0.374 & 0.070 & 0.750 & 0.064 \\
\hline Theory $^{b}$ & AlAs & 0.413 & 0.183 & 1.136 & 0.143 \\
2nn (this work) & AlAs & 0.416 & 0.178 & 1.137 & 0.140 \\
1 nn (this work) & AlAs & 0.605 & 0.134 & 1.136 & 0.122 \\
\hline
\end{tabular}

a Reference 10

${ }^{b}$ Reference 11

In order to investigate the possibility that small uncertainties in the effective masses for AlAs might produce better agreement with experiment $\mathrm{Tthe}$ sensitivity of the binding energy to the $\mathrm{HH}$ masses is studied. The
HH masses for AlAs are scaled by a factor $\tilde{m}$ ranging from 0.60 and 1.40 in such a way as to not modify any of the band energies at $\Gamma$ [9]. Even though scaling the $\mathrm{HH}$ masses by such a large fraction may have an 
adverse effect on some conduction-band features $\Gamma$ these are of little importance in determining acceptor binding energies. In the inset of Fig.1Tthe acceptor energy at $x=0.4$ is plotted as a function of $\tilde{m}$ Tfor both $1 \mathrm{nn}$ and $2 \mathrm{nn}$ cases. A remarkably linear behavior is observed over a wide range of $\mathrm{HH}$ masses. Interestingly the $1 \mathrm{nn}$ model is much more sensitive to variations in the $\mathrm{HH}$ mass $\Gamma$ with even a modest increase greatly improving agreement with experiment. For the $2 n n$ modelThoweverTeven increasing the HH mass by $40 \%$ leads to binding energy of only 93 meVTcompared to $120 \mathrm{meV}$ in experiment. It is worthwhile here to comment that in order to obtain good agreement with experiment (say $110 \mathrm{meV}$ at $x=0.4$ ) a binding energy of $\sim 400 \mathrm{meV}$ is required for $x=1.0$ (i.e. Tpure AlAs). This energy serves as a useful reference for the analysis considered below.

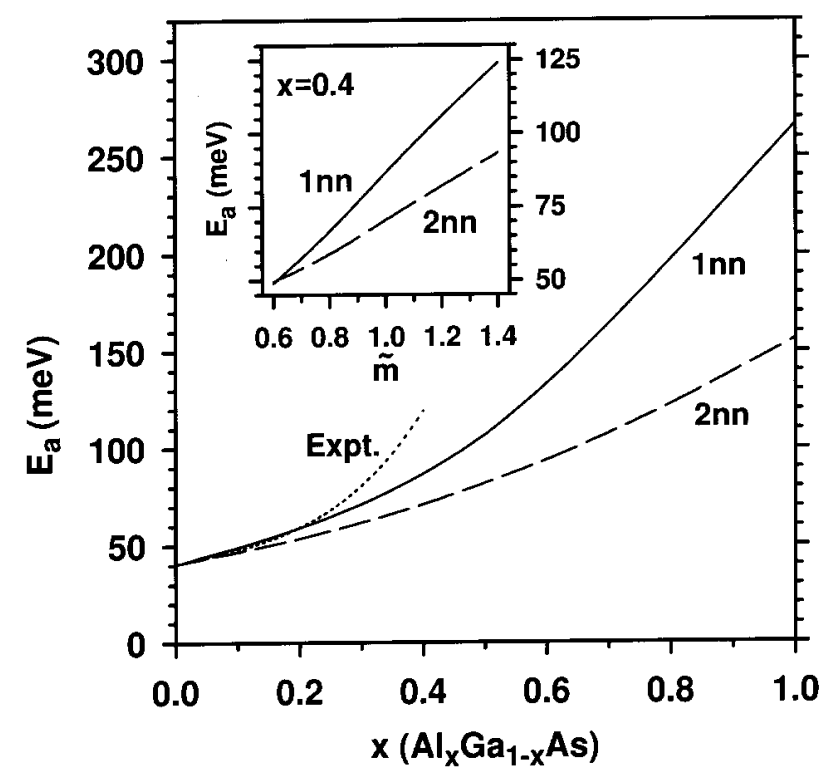

Figure 1. Acceptor energies $E_{a}$ for Ge impurities in $\mathrm{Al}_{x} \mathrm{Ga}_{1-x}$ As alloys, calculated with $1 \mathrm{nn}$ (solid line) and $2 \mathrm{nn}$ parametrizations (dashed line). The experimental result is given by the dotted line. Inset: acceptor energy at $x=0.4$ as a function of the mass scaling factor $\tilde{m}$ for 1 nn and $2 \mathrm{nn}$ parametrizations.

Varying the TB parameters that determine the $\mathrm{HH}$ masses in effect changes the bulk properties of the host. HoweverTit is also possible to vary the TB parameters that pertain only to the impurity $\Gamma$ without modifying the bulk properties. These parameters would therefore describe central-cell effects $\Gamma$ and can be incorporated within the present theory through the on-site energy $U_{0}$ of the impurity itself. Up to now Tit has been assumed that $U_{0}$ was independent of the host. Nevertheless Tit is instructive to consider what effect variations in $U_{0}$ have on the impurity energies. In Fig.2(a) the acceptor en- ergy calculated for a range of $U_{0}$ is presented for GaAs and AlAs using the $2 \mathrm{nn}$ parametrizations. The curves are characterized by three distinct regimes: an energetically flat regime for small $U_{0} \Gamma$ a linearly increasing regime for large $U_{0}$ Tand a transition region for intermediate $U_{0}$. In the flat region $\Gamma$ different impurity species (characterized by different $U_{0}$ ) will have nearly identical binding energies $\Gamma$ meaning that central-cell corrections will be very small. Such behavior is typical of shallow levelsTwhich implies that EMT is expected to work well in this regime.

It is interesting to note that in going from GaAs to AlAs (i.e. Tincreasing HH mass) $\mathrm{H}$ the energetically flat regime gets pushed to ever smaller $U_{0}$. In fact Tfor AlAsT the flat region is never quite reached Texcept perhaps for $U_{0}<1 \mathrm{eV}$ using $2 \mathrm{nn}$ Tand so central-cell corrections are expected to always be important for this host. It should also be kept in mind that the Coulomb potential due to the impurity as given by Eq. (2) is roughly $0.6 \mathrm{eV}$ at the nearest-neighbor distance $(2.45 \AA)$. Therefore $\Gamma$ it is unphysical to consider acceptors with $U_{0}$ smaller than this value. The linear regime observed for large $U_{0}$ marks the breakdown of the effective mass approach. In EMTT the impurity potential is assumed to be slowly varying on the atomic length scale. A potential of say $5.0 \mathrm{eV}$ at the impurity site $\Gamma$ falling to $0.6 \mathrm{eV}$ at the nearest neighbor $\Gamma$ clearly violates this approximation. In the linear regime $\Gamma$ central-cell effects are expected to be very important Tbecause even small variations in $U_{0}$ (due to different impurities) will lead to large changes in binding energy.

In Fig.2(b) the impurity energy is plotted as a function of $U_{0}$ for AlAs and GaAs using the $1 \mathrm{nn}$ parametrization. QualitativelyT the results are similar to the $2 \mathrm{nn}$ case shown in Fig. (a). However $\Gamma$ the $1 \mathrm{nn}$ case is once again found to be far more sensitive than the $2 \mathrm{nn}$ case. For instance Tincreasing $U_{0}$ to just $3.3 \mathrm{eV}$ is sufficient to give a binding energy of $400 \mathrm{meV}$ in AlAs with $1 \mathrm{nn}$ Twhereas a value of $5.5 \mathrm{eV}$ is required with a 2nn model. Recalling that for GaAs the value of $U_{0}$ was $3.42 \mathrm{eV}$ for $2 \mathrm{nn}$ and $2.80 \mathrm{eV}$ for $1 \mathrm{nn} \Gamma$ and considering the chemical similarity between GaAs and AlAs a value of $5.5 \mathrm{eV}$ seems unrealistic. Therefore $\Gamma$ the interpretation once more is that the $1 \mathrm{nn}$ results are more consisitent with experiment.

In order to obtain a better understanding of the connection between $1 \mathrm{nn}$ and $2 \mathrm{nn}$ models $\Gamma$ it is necessary to compare the two cases directlyTusing the same value of $U_{0}$. According to EMTT the acceptor binding 
energy should depend only on the effective masses near the $k$ point under consideration. From this point of view $\Gamma$ no difference should exist between $1 \mathrm{nn}$ and $2 \mathrm{nn}$ parametrizations $\Gamma$ as long as the effective masses were the same. In order to quantitatively study the validity of this assertion Thowever Tthe $1 \mathrm{nn}$ and $2 \mathrm{nn}$ TB parameters must be adjusted so as to give the same HH masses in the (100) as well as (111) directions [9]. NextTa value of $U_{0}$ sufficiently small $(0.7 \mathrm{eV})$ is chosen so that the EMT should be expected to hold Tand the binding energy calculated as a function of $x$ Twhere as usual $x$ is the Al concentration of the $\mathrm{Al}_{x} \mathrm{Ga}_{1-x}$ As alloy. The results are plotted in Fig.3 5 and show that the $1 \mathrm{nn}$ and $2 \mathrm{nn}$ results are in almost exact agreement over the entire range of $x$. This is reassuring $\Gamma$ because for shallow levels the energy can only depend on the effective mass if the TB result is to be consisitent with EMT. The second case to be considered is for larger $U_{0} \Gamma$ in which the EMT can be expected to break down. A value of $U_{0}=3.5 \mathrm{eV}$ is used and the energy $E_{a}(x)$ recalculated. From Fig. $3 \Gamma$ a radical difference is now observed between $1 \mathrm{nn}$ and $2 \mathrm{nn}$ parametrizations. For GaAsT the $1 \mathrm{nn}$ result gives $70 \mathrm{meV}$ compared with only $42 \mathrm{meV}$ using $2 \mathrm{nn}$. In passing $\mathrm{Tit}$ is instructive to note that the EMT result breaks down strongly for the $U_{0}=3.5 \mathrm{eV}$ case in GaAs but not for the $U_{0}=0.7 \mathrm{eV}$ case in AlAs even though the binding energies are comparable. This demonstrates that binding energy considerations alone are not enough to determine if a level can or cannot be described by EMT. In any case $\Gamma$ the breakdown of EMT is even more dramatic for the $U_{0}=3.5 \mathrm{eV}$ case in pure AlAs $\Gamma$ with the $1 \mathrm{nn}$ approach yielding an energy of $454 \mathrm{meV}$ compared with $182 \mathrm{meV}$ using $2 \mathrm{nn}$. The experimental data suggest $\Gamma$ therefore $\Gamma$ that 1 inn descriptions are more appropriate than $2 n n$ for the case of deep levels. The likely reason is that the $2 \mathrm{nn}$ hopping terms permit the electron to escape more easily from the impurity potential Tthereby delocalizing the wave function and reducing the binding energy. To confirm this conjecture $\Gamma$ the radial charge distribution is calculated for an AlAs host using both $1 \mathrm{nn}$ and $2 \mathrm{nn}$ with an on-site energy $U_{0}$ of $3.5 \mathrm{eV}$. It is found indeed that the electron is far more delocalized using the $2 \mathrm{nn}$ approach. For instance using $1 \mathrm{nn}$ There is a $90 \%$ probability of localizing the electron within $7 \AA$ of the impurity $\Gamma$ whereas using the $2 \mathrm{nn}$ parametrization The same probability is reached at roughly $14 \AA$.

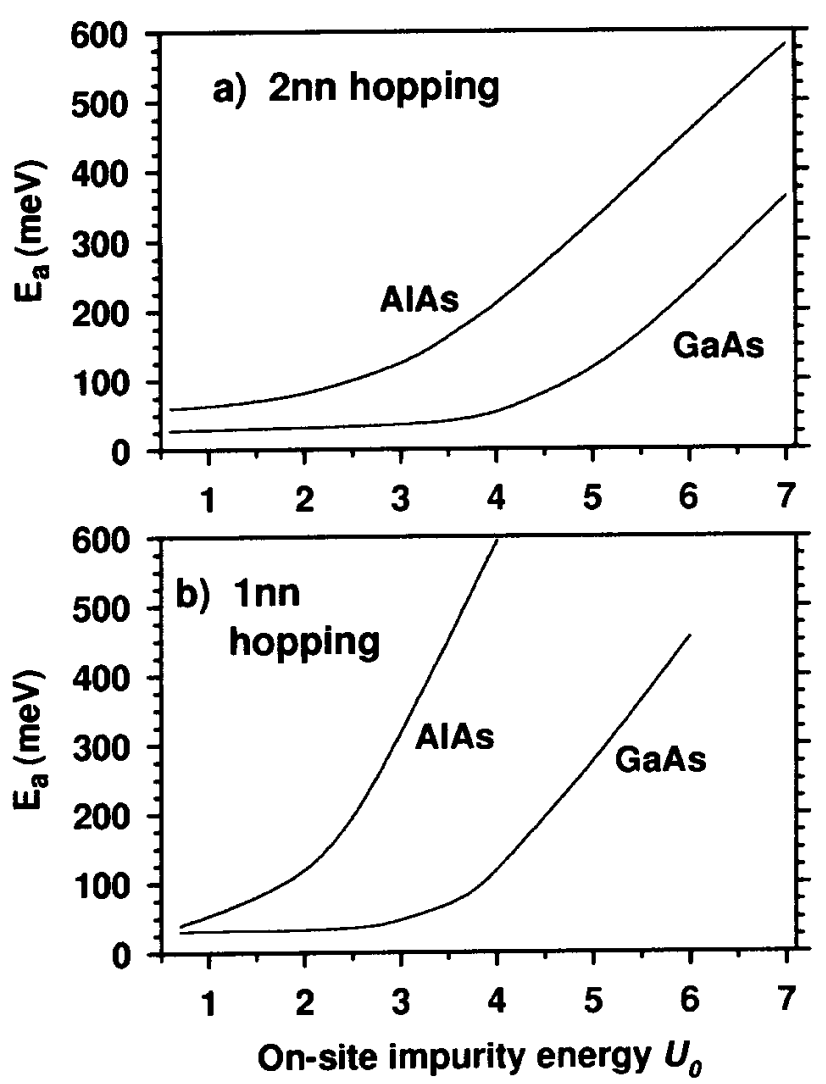

Figure 2. Acceptor binding energies for Ge impurities as a function of the on-site impurity potential $U_{0}$ (a) Using $2 \mathrm{nn}$ parametrization for AlAs and GaAs. (b) Using 1nn parametrization for AlAs and GaAs. The flat region for small $U_{0}$ indicates the regime in which EMT is expected to work well.

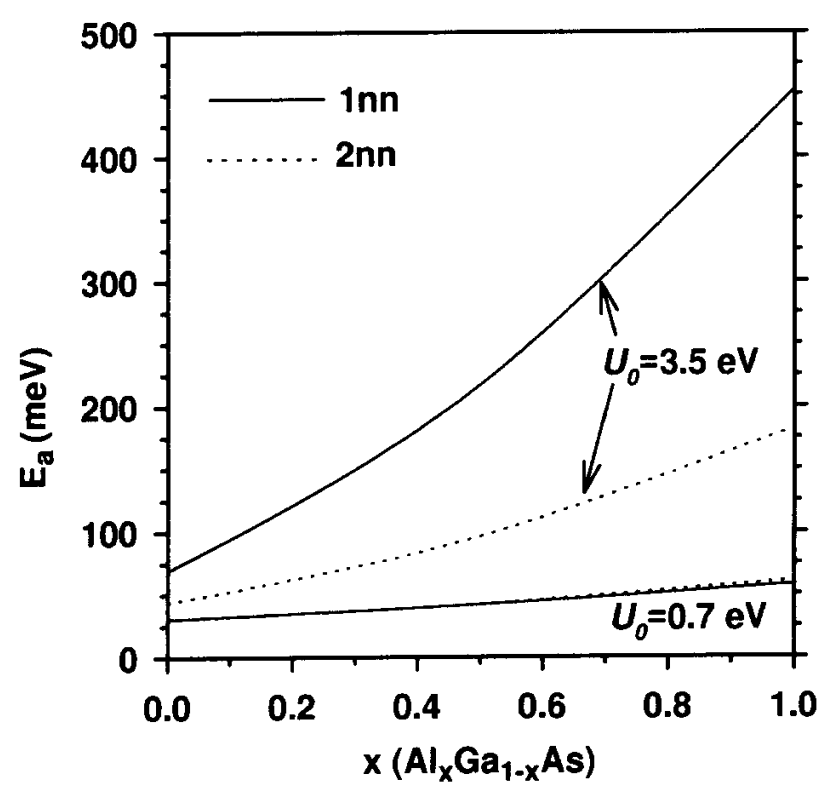

Figure 3. Acceptor energies calculated for shallow levels $\left(U_{0}=0.7 \mathrm{eV}\right)$ and deep levels $\left(U_{0}=3.5 \mathrm{eV}\right)$ using $1 \mathrm{nn}$ (solid line) and $2 \mathrm{nn}$ (dotted line) parametrizations, with the same $H H$ effective masses. The $U_{0}=0.7$ results are consistent with EMT, whereas major deviations are observed for $U_{0}=3.5$. 


\section{Summary and Conclusions}

Binding energies have been calculated for acceptors in GaAsTAlAsTand $\mathrm{Al}_{x} \mathrm{Ga}_{1-x}$ As hosts. It was found that the $1 \mathrm{nn}$ model is much more sensitive than the $2 \mathrm{nn}$ model to small changes in the parameters. Insight was gained into why and when central-cell corrections become important. For shallow levels in which central-cell corrections are unimportant $\Gamma$ the $1 \mathrm{nn}$ and $2 \mathrm{nn}$ models give the same results. HoweverT for intermediate to deep levels $\Gamma$ the two results deviate $\Gamma$ and experimental evidence suggests that the $1 \mathrm{nn}$ model is more appropriate. The reason is that the $2 \mathrm{nn}$ model is not as effective in binding the wave function to the impurity. The $2 \mathrm{nn}$ parametrizations are expected to be most useful therefore for describing shallow levelsT due to their superior ability to fit the effective masses. A particularly interesting application of the $2 \mathrm{nn}$ approach may be for the case of donors in indirect gap materials $\Gamma$ due to intrinsic limitations in the $1 \mathrm{nn}$ ability to fit conduction- band dispersion near the zone boundary.

\section{Acknowledgements}

Helpful discussions with Belita KoillerTRodrigo CapazT Helio Chacham $\Gamma$ and Timothy Boykin are gratefully acknowledged. The financial support of $\mathrm{CNPq} \Gamma$ PRONEXTand FINEP is also greatly appreciated.

\section{References}

[1] C. Kittel and A. H. Mitchell, Phys. Rev. 96, 1488 (1954).

[2] J. M. Luttinger, and W. Kohn, Phys. Rev. 97, 869 (1955).

[3] C. Kittel, Introduction to Solid State Physics, 7th Ed., (Wiley, 1996) p. 225.

[4] J. G. Menchero, R. B. Capaz, Belita Koiller, and H. Chacham, Phys. Rev. B 59, 2722 (1999).

[5] P. Vogl, H. P. Hjalmarson, and J. D. Dow, J. Phys. Chem. Solids 44, 365 (1983).

[6] J. Bernholc and S. T. Pantelides, Phys. Rev. B 15, 4935 (1977)

[7] J. K. L. MacDonald, Phys. Rev. 46, 828 (1934); R. B. Capaz, G. C. de Araujo, Belita Koiller, and J. P. von der Weid, J. Appl. Phys. 745531 (1993); L. W. Wang and A. Zunger, J. Chem. Phys. 100, 2394 (1994).

[8] R. Enderlein, G. M. Sipahi, L. M. R. Scolfaro, and J. R. Leite, Phys. Stat. Sol. (b) 206, 623 (1998).

[9] J. G. Menchero and T. B. Boykin, Impurity states in semiconductors calculated via tight-binding: a parameter sensitivity study, Phys. Rev. B 59, to appear (1999).

[10] R. V. Shanabrook, O. J. Glembocki, D. A. Broido, and W. I. Wang, Phys. Rev. B 39, 3411 (1989).

[11] L. Pavesi and M. Guzzi, J. Appl. Phys. 75, 4779 (1994).

[12] G. Oelgart, B. Lippold, R. Heilmann, H. Neumann, and B. Jacobs, Phys. Stat. Sol. A 115, 257 (1989). 